\title{
A lateral approach to exchange a left ventricular assist device
}

\author{
Jasmin S. Hanke, MD, Günes Dogan, MD, and Jan D. Schmitto, MD, PhD, MBA, FCCP, FRCS (Glasgow), \\ Hannover, Germany
}

\footnotetext{
From the Department of Cardiac, Thoracic, Transplantation, and Vascular Surgery, Hannover Medical School, Hannover, Germany.

Disclosures: J.D.S. and G.D. are consultants for Abbott Laboratories. J.S.H. have nothing to disclose with regard to commercial support.

No funding was provided for this work

Received for publication March 28, 2018; revisions received Dec 14, 2018; accepted for publication Dec 25, 2018; available ahead of print Feb 20, 2019.

Address for reprints: Jan D. Schmitto, MD, PhD, MBA, FCCP, FRCS (Glasgow), Department of Cardiac, Thoracic, Transplantation and Vascular Surgery, Hannover Medical School, Carl-Neuberg Strasse 1, 30625 Hannover, Germany (E-mail: Schmitto.Jan@mh-hannover.de).

J Thorac Cardiovasc Surg 2019;158:e39-40

$0022-5223 / \$ 36.00$

Copyright (c) 2019 by The American Association for Thoracic Surgery

https://doi.org/10.1016/j.jtcvs.2018.12.081
}

Since its release, the HeartMate 3 (HM3; Abbott Laboratories, Abbott Park, Ill) has been considered a left ventricular assist device (LVAD) with a low risk for thromboembolic events such as pump thrombosis. ${ }^{1-3}$ Thus far, no pump thromboses for the HM3 have been described. In this report, we describe HM3 exchange for pump thrombosis likely caused by ingestion of the preexisting thrombus of the left atrium (Figure 1).

We present the case of a 64-year-old patient who underwent less-invasive HM3 implantation because of dilated cardiomyopathy in November 2015. The implantation was performed through an anterolateral thoracotomy and ministernotomy. ${ }^{3,4}$ The initial postoperative course was uneventful, and the patient was successfully discharged to rehabilitation. In March 2016, the patient had a severe driveline infection and was listed for heart transplantation. A computed tomographic scan was performed and showed a newly formed left atrial thrombus that was not present before LVAD implantation (Figure 1). Despite the detected thrombus, we did not act on the new finding because of the sound clinical status of the patient and the prospect of cardiac transplantation. In November 2016, however, the patient had a thromboembolic LVAD occlusion occur, with recurrent high-flow- and low-flow alarms as well as hematuria and a deterioration of his overall condition. International normalized ratio measurements did not show any irregularities (range, 1.83-3.66). The former thrombus in the left atrium was not detectable with echocardiography. The patient was scheduled for urgent LVAD exchange and gave informed consent for the procedure.

The access point for the procedure was determined by echocardiography. A lateral thoracotomy was performed in the sixth intercostal space. Partial resection of one rib was necessary to gain access to the pump corpus. The

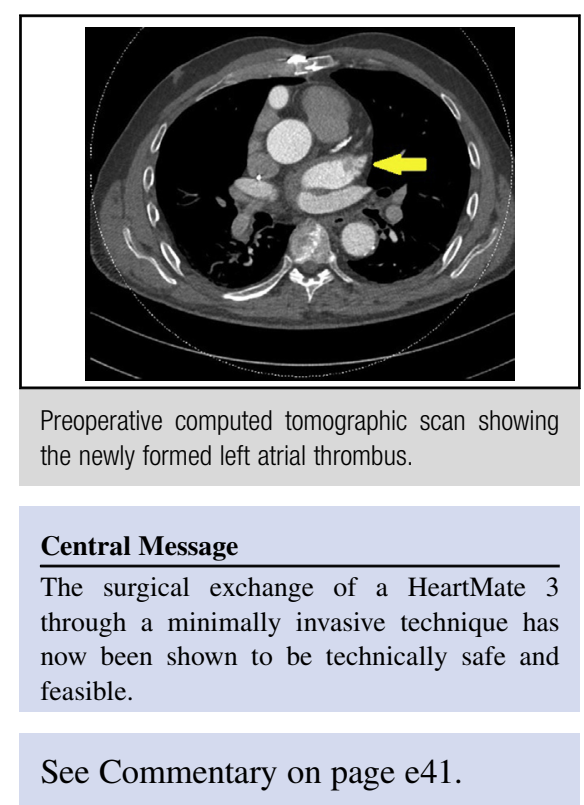

venous and the arterial lines of the heart-lung machine were placed in the femoral artery and vein. After exposition of the pump position, the outflow graft was clamped, and the HM3 was turned off and removed from the thorax. After removal of the pump housing from the old sewing ring, the left ventricular cavity was carefully inspected, and any remaining thrombus residences were completely removed. Intracavitary thrombotic deposits, as well as deposits inside the pump housing, were discovered.

Setup of the new HM3 was performed according to standard instructions for use of the company protocol. Next, the new pump was placed in the old sewing ring. The suction of the heart-lung machine was placed into the outflow graft of the HM3 after deairing of the left ventricle and the pump. Thereafter, the new device was started, and the driveline was tunneled through the abdominal wall by standard technique. The old outflow graft was still located within the thorax, and an anastomosis between the remaining outflow graft of the old HM3 and the outflow graft prosthesis of the new HM3 was performed. To ensure hemostasis during LVAD exchange, the procedure was performed with the use of red blood cells, fresh-frozen plasma, thrombocytes, and coagulation factors.

The patient was in hemodynamically stable condition and was extubated on postoperative day 1 . The patient's further postoperative course was uneventful. He was 


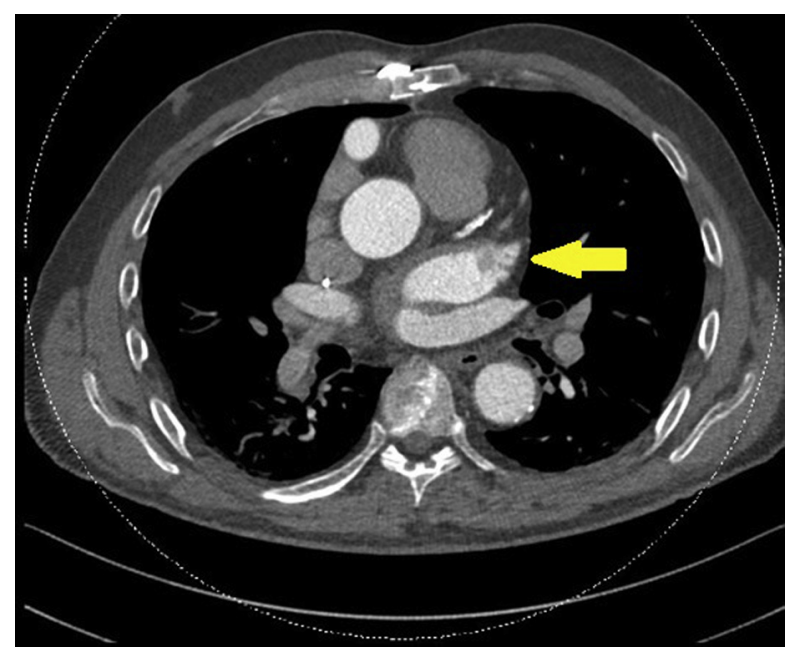

FIGURE 1. Preoperative computed tomographic scan demonstrating the newly formed left atrial thrombus (arrow).

successfully discharged to a rehabilitation center and is now awaiting heart transplantation.

Even though existing literature indicates extremely low thromboembolic occlusion rates of the HM3 LVAD, ${ }^{1-3}$ this case report represents the first description of an exchange of a thromboembolic occluded device. As a limiting factor, however, it has to be considered that the preoperatively presented thrombus may likely have been the cause for pump thrombosis, rather than the de novo thrombus formation in the pump corpus. To this end, the diligent inspection of the left ventricular cavity at the time of LVAD implantation is of major importance to avoid possible thrombotic events.

Evidently, HM3 exchange can be easily and safely performed through an anterolateral thoracotomy. ${ }^{4,5}$ Furthermore, it is technically possible to perform the procedure without the use of a heart-lung machine. Because of the high risks of stroke and air embolism, however, we refrain from using off-pump techniques for this procedure, at least as long as there are no tools to prevent the associated complications.

As shown in this report, the surgical exchange of a HM3 through a lateral thoracotomy has proved to be a technically safe and feasible procedure.

\section{References}

1. Schmitto JD, Hanke JS, Rojas SV, Avsar M, Haverich A. First implantation in man of a new magnetically levitated left ventricular assist device (HeartMate III). $J$ Heart Lung Transplant. 2015;34:858-60.

2. Mehra MR, Naka Y, Uriel N, Goldstein DJ, Cleveland JC Jr, Colombo PC, et al; MOMENTUM 3 Investigators. A fully magnetically levitated circulatory pump for advanced heart failure. $N$ Engl J Med. 2017;376:440-50.

3. Netuka I, Sood P, Pya Y, Zimpfer D, Krabatsch T, Garbade J, et al. Fully magnetically levitated left ventricular assist system for treating advanced HF: a multicenter study. J Am Coll Cardiol. 2015;66:2579-89.

4. Schmitto JD, Rojas SV, Hanke JS, Avsar M, Haverich A. Minimally invasive left ventricular assist device explantation after cardiac recovery: surgical technical considerations. Artif Organs. 2014;38:507-10.

5. Rojas SV, Avsar M, Khalpey Z, Hanke JS, Haverich A, Schmitto JD. Minimally invasive off-pump left ventricular assist device exchange: anterolateral thoracotomy. Artif Organs. 2014;38:539-42. 